\title{
Tuberculous Meningitis and COVID-19 Coinfection: A Diagnostic Challenge
}

\author{
Sara S Dhanawade ${ }^{1}$, Aditya V Kurade ${ }^{2}$
}

\begin{abstract}
The neurological manifestations of coronavirus disease-2019 (COVID-19) infection in children is not well documented. We hereby report a case of tuberculous meningitis and COVID-19 coinfection complicated by hydrocephalus and multiple arterial infarcts. The overlapping clinical presentation posed a diagnostic and therapeutic challenge.
\end{abstract}

Keywords: Arterial infarcts, Coronavirus disease-2019, Hydrocephalus, Tuberculous meningitis.

Pediatric Infectious Disease (2021): 10.5005/jp-journals-10081-1299

\section{INTRODUCTION}

Neurological complications of coronavirus disease-2019 (COVID-19) are being increasingly reported in adults. Seizures, encephalopathy, acute necrotizing encephalitis, stroke, and GBS are reported in adults. ${ }^{1}$ However, COVID-19 infection is typically mild in children and no neurological complications were reported in the 171 children from Wuhan. ${ }^{2}$ Pediatric TBM and COVID-19 co-infection was first reported by Essajee et al. ${ }^{3}$ Our case also had TBM and COVID-19 co-infection and highlights the challenges associated with coinfections with similar clinical presentations.

\section{Case Description}

A 4-year-old previously well child presented with fever, abdominal pain of 8 days followed by seizures, and altered sensorium of 2 days. There was no history of household contact either with TB or COVID-19. She was poorly nourished and belonged to a low socioeconomic family. She was fully immunized and BCG scar was present. There was no history of prior treatment elsewhere. At admission, the child was unresponsive (GCS-3), pupils were equal, semi-dilated but sluggishly reacting. The vital signs were stable. She had decerebrate posturing, brisk DTRs, and extensor plantar bilaterally. Other systems were normal. She was immediately intubated and ventilated. Investigations revealed $\mathrm{Hb}-9.4 \mathrm{~g} / \mathrm{dL}$, TLC-11.5 $\times 10^{3} / \mathrm{L}$ (neutrophils-91\%, lymphocytes-4\%), platelets-349 $\times 10^{9} / \mathrm{L}$, ESR-20 mm/hour, Q-CRP-110 mg/L, chest $\mathrm{X}$-ray was normal. The liver, kidney functions were normal and INR was 1.4. CT brain with contrast showed left temporal hypodensities, mild ventriculomegaly, and generalized cerebral edema. CSF examination revealed 170 cells $/ \mathrm{mm}^{3}$ (neutrophils- $2 \%$, lymphocytes-98\%), protein $25 \mathrm{mg} / \mathrm{dL}$ and glucose $10 \mathrm{mg} / \mathrm{dL}$ (blood sugar-124 mg/dL). The nasopharyngeal swab was positive for SARSCoV-2. A diagnosis of SARS-CoV-2 associated meningoencephalitis was made. The possibility of tuberculous meningitis was considered in view of the CSF and CT scan picture. CSF smear and CBNAAT were negative for AFB. The inflammatory markers revealed IL-6 $13.6 \mathrm{pg} / \mathrm{mL}$, D-dimer $9.8 \mathrm{mg} / \mathrm{L}$, Sr.ferritin $100 \mathrm{ng} / \mathrm{mL}$, and LDH $932 \mathrm{IU} / \mathrm{L}$. CSF for SARS-CoV-2 could not be done as a facility for the same was not available. The encephalitis PCR panel on CSF for Japanese encephalitis, herpes simplex, and Chandipura virus were negative. CSF India ink preparation was negative and the
1,2Department of Pediatrics, Bharati Vidyapeeth (Deemed to be University) Medical College and Hospital, Sangli, Maharashtra, India

Corresponding Author: Sara S Dhanawade, Department of Pediatrics, Bharati Vidyapeeth (Deemed to be University) Medical College and Hospital, Sangli, Maharashtra, India, Phone: +91 9890936995, e-mail: sarasubodh@yahoo.com

How to cite this article: Dhanawade SS, Kurade AV. Tuberculous Meningitis and COVID-19 Coinfection: A Diagnostic Challenge. Pediatr Inf Dis 2021;3(2):79-80.

Source of support: Nil

Conflict of interest: None

culture was sterile. She was managed with ceftriaxone, intravenous fluids, mannitol, anticonvulsants, dexamethasone, and intravenous immunoglobulin. She received remdesivir for 5 days under the compassionate use protocol. She was given enoxaparin for 10 days in view of elevated D-dimer. She continued to have multiple seizures and required fosphenytoin, levetiracetam, and phenobarbitone. Convulsions were controlled after 48 hours. CSF analysis repeated after 48 hours showed 58 cells $/ \mathrm{mm}^{3}$ (polymorphs $2 \%$, lymphocytes 98\%), protein $20 \mathrm{mg} / \mathrm{dL}$, glucose $20 \mathrm{mg} / \mathrm{dL}$ (blood sugar $150 \mathrm{mg} /$ $\mathrm{dL}$ ). The nasopharyngeal RT-PCR for SARS-CoV-2 repeated on day 8 was negative. MRI brain could be done only on the 8th hospital day which showed multiple infarcts in the right capsuloganglionic region, right occipital cortical-subcortical area, left ganglionic region, midbrain, dilated third and fourth ventricles, and significant basal meningeal enhancement.

She remained comatose with minimal response to painful stimuli by flexion. The CSF examination repeated after 8 days showed total WBC 97 cells $/ \mathrm{mm}^{3}$ (polymorphs 30\%, lymphocyte $70 \%$ ), protein $43 \mathrm{mg} / \mathrm{dL}$, glucose $50 \mathrm{mg} / \mathrm{dL}$ (blood sugar $127 \mathrm{mg} / \mathrm{dL}$ ). As the clinical presentation, CSF and MRI findings strongly favored tuberculous meningitis she was started on 4 drug antitubercular treatment (ATT) on day 9th of admission, and dexamethasone was continued. She was weaned off the ventilator on the 14th day. Her sensorium started improving after 10 days of ATT, although she had prolonged fever. MRI repeated showed old infarct with gliosis, dilated third and fourth ventricle. CSF study after 4 weeks of ATT 
still showed $90 \mathrm{WBCs} / \mathrm{mm}^{3}$ (polymorphs 40\%, lymphocytes $60 \%$ ), glucose $60 \mathrm{mg} / \mathrm{dL}$ (blood sugar $86 \mathrm{mg} / \mathrm{dL}$ ), and protein $75 \mathrm{mg} / \mathrm{dL}$.

During the hospital stay, she received intensive neurorehabilitation therapy and was discharged after 6 weeks on nasogastric feeds with advice to continue physiotherapy. At discharge, she had left hemiparesis, generalized spasticity, poor head control, and no speech.

\section{Discussion}

It is not clear whether SARS-CoV-2 directly invades the nervous system. The first case of COVID-19 encephalitis was reported by McAbee et al. in an 11-year-old child who recovered completely within 6 days. ${ }^{4}$ There is one pediatric case report from India of acute encephalopathy in COVID-19 infection. ${ }^{5}$ Confirming the diagnosis of TBM in children is difficult. Our patient presented with acute onset of symptoms. Nevertheless, the CSF picture showed lymphocytic pleocytosis with hypoglycorrhachia and imaging studies revealed the classic triad of hydrocephalus, infarctions, and basal meningeal enhancement. The patient had a protracted clinical course with prolonged fever, persistent CSF pleocytosis, and significant neurocognitive sequelae despite adequate treatment. Hydrocephalus and vasculitis delay the resolution of symptoms of TBM. ${ }^{6}$ The difficulty in distinguishing overlapping clinical presentation particularly in the acute stage delayed the diagnosis and initiation of ATT. Both TBM and COVID-19 infection induce a proinflammatory response resulting in an increased risk of venous thrombosis and ischemic stroke. In the first reported case, the child had extensive cerebral sinus thrombosis in addition to an arterial ischemic stroke which was attributed to COVID-19 co-infection. ${ }^{3}$ Our patient also had elevated D-dimer and it is possible that COVID-19 co-infection contributed to the hypercoagulable state and severe clinical evolution.

While dealing with COVID-19 cases presenting with symptoms of meningitis or encephalitis, one should not forget TB as a coexisting pathology in countries like India. Although our patient had TBM with vasculitic complications, presentation during the COVID-19 pandemic resulted in diagnostic dilemma. This case provides an important message of clinical medicine, that common manifestations of common diseases should be identified first. A timely review and optimal evaluation remain the mainstay in making the correct diagnosis.

\section{References}

1. Mao L, Jin $H$, Wang $M$, et al. Neurological manifestations of hospitalized patients with coronavirus disease 2019 in Wuhan, China. JAMA Neurol 2020;77(6):683-690. DOI: 101001/jamaneurol20201127.

2. Lu X, Zhang $\mathrm{L}$, Du H, et al. SARS-CoV-2 infection in children. $\mathrm{N}$ Engl J Med 2020;382(17):1663-1665. DOI: 10.1056/NEJMc2005073.

3. Essajee F, Solomons R, Goussard P, et al. Child with tuberculous meningitis and COVID-19 coinfection complicated by extensive cerebral sinus venous thrombosis. BMJ Case Rep CP 2020;13(9):e238597. DOI: 10.1136/bcr-2020-238597.

4. McAbee GN, Brosgol Y, Pavlakis S, et al. Encephalitis associated with COVID-19 infection in an 11-year-old child. Pediatr Neurol 2020;109:94. DOI: 10.1016/j.pediatrneurol.2020.04.013.

5. Kinikar A, Kulkarni R, Rajput U, et al. Acute encephalopathy in a child with coronavirus disease-2019 infection. Pediatr Inf Dis 2020;2(2):6263.

6. Pradhan S. Hydrocephalus and vasculitis delay therapeutic responses in tuberculous meningitis: the neurologist's perspective. Neurol India 2016;64(5):860-861. DOI: 10.4103/0028-3886.190293. 